\title{
Neurosurgical Economic and Readmission Trends After Extracranial Ventricular Shunts in the United States From 2009 to 2013
}

\author{
Ross-Jordon S. Elliott ${ }^{\mathrm{a}, \mathrm{d}}$, Marwah A. Elsehety ${ }^{\mathrm{b}}$, Ali Seific
}

\begin{abstract}
Background: The aim of the study was to define the association between federal payer insurance and neurosurgical economic trends and readmissions after extracranial ventricular shunts (EVS) procedures and investigate these trends from 2009 to 2013 in the United States.

Methods: We identified the procedure of insertion, replacement, or removal of EVS by applying the International Classification of Disease, Ninth Edition, Clinical Modification (ICD-9-CM) Procedure Codes of 231-235, 239, 242 and 243. Data were extracted for years 2009 to 2013. Year-wise distributions of index stays, readmission, percent readmission, cost for index stays and cost for readmissions for patients requiring EVS procedures who possess Medicare insurance (ME-patients) and Medicaid insurance (MD-patients) were described. Z-test statistic was used to compare the two groups.
\end{abstract}

Results: During the 5 years of study, we recorded 149,220 index stays and 29,655 readmissions within 30 days involving the procedures of insertion, replacement, or removal of an EVS. Throughout the study period, hospital readmissions involving patients requiring procedures involving EVS consistently demonstrated both the highest annual mean cost for readmissions and the highest percentage of patient readmissions in regard to all neurosurgical procedures. The differences between the annual index stays and readmissions for MEpatients versus MD-patients requiring EVS were extremely statistically significant throughout the entire study period $(\mathrm{P}<0.0001, \mathrm{P}<$ $0.0001)$. The mean cost of readmissions within 30 days for all patients varied significantly from $\$ 19,005$ to $\$ 23,499$, with an average cost of $\$ 21,279$ for readmissions occurring annually during the study period

Manuscript submitted May 19, 2020, accepted May 25, 2020

Published online June 3, 2020

aDepartment of Neurological Surgery, The George Washington University Hospital, Washington, DC, USA

bDepartment of Neurology, University of Texas Health Science Center at Houston, Houston, TX, USA

'Department of Neurosurgery and Neurology, University of Texas Health Science Center at San Antonio, San Antonio, TX, USA

${ }^{\mathrm{d} C o r r e s p o n d i n g ~ A u t h o r: ~ R o s s-J o r d o n ~ S . ~ E l l i o t t, ~ D e p a r t m e n t ~ o f ~ N e u r o l o g i-~}$ cal Surgery, The George Washington University Hospital, 900 23rd St. NW, Washington, DC 20037, USA. Email: Relliott1@gwu.edu

doi: https://doi.org/10.14740/jnr600
$(\mathrm{P}=0.0161)$. The differences between the mean cost for index stays and readmissions for ME-patients versus MD-patients requiring EVS were extremely statistically significant throughout the entire study pe$\operatorname{riod}(\mathrm{P}<0.0001, \mathrm{P}<0.0001)$.

Conclusions: Federal payer insurance has a significant association with neurosurgical economic and patient readmission trends after EVS procedures in hospitals in the US. Further study is needed to investigate the etiology of these differences between patients' payer insurance and their impact on clinical outcomes after EVS procedures.

Keywords: Payer insurance; Extracranial ventricular shunts; Readmissions; Index stays; Economics

\section{Introduction}

External ventricular drains (EVD) or extracranial ventricular shunts (EVS) are necessary in many neurosurgical emergencies and in the treatment of diseases such as hydrocephalus. Procedures involving EVS, however, are not without their risks which include surgical site or cerebrospinal fluid (CSF) infections, intracranial or intraventricular hemorrhage, seizure, neurological deficits, coma and death $[1,2]$. Due to the incidence of these complications and the growing economic cost of healthcare, the rate of readmissions after EVS procedures is of particular interest to both neurosurgeons and hospital administrators, as ventricular shunts remain one of the most commonly performed neurosurgical procedures resulting in a significant monetary cost from a national perspective $[1,2]$. Given the previously reported significant medical and economic quandary that EVS procedures pose to the medical community and patients in prior registry studies, we investigated whether this association was evident in a large national database throughout the entire population $[1,2]$. There is a paucity of information on these trends in the literature. We sought to examine the rate of readmission and economic trends across different payer groups after EVS procedures in American hospitals. Utilizing data from the Nationwide Inpatient Sample (NIS) of the Healthcare Cost and Utilization Project (HCUP), we analyzed the association between federal payer insurance and neurosurgical economic trends and readmissions after EVS procedures and investigated these trends from 2009 to 2013 in the United States. 


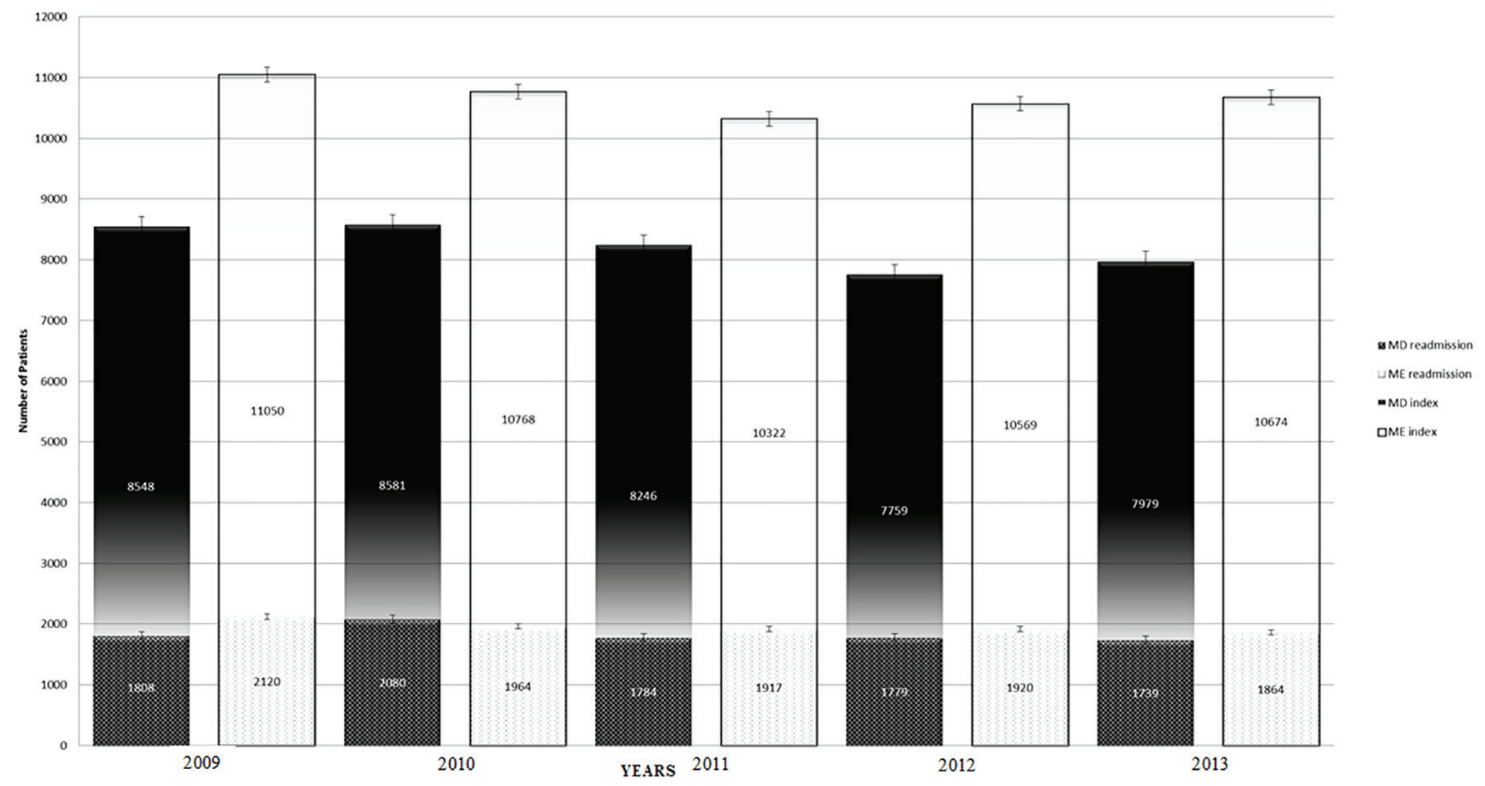

Figure 1. Clustered bar graph of Medicare and Medicaid index stays and readmissions after extracranial ventricular shunt (EVS) procedures annually from 2009 to 2013 . The differences between the annual index stays and readmissions for Medicare (ME) and Medicaid (MD) patients requiring EVS were extremely statistically significant throughout the entire study period $(P<0.0001$, $P<0.0001)$.

\section{Materials and Methods}

For our study, we conducted a retrospective analysis utilizing data obtained from the NIS, which was gathered by the HCUP network (HCUPnet), which collects hospital discharge information from non-federal, short-term, general and specialty-specific hospitals [3]. The NIS represents approximately a $20 \%$ stratified sample of the hospitals represented in the HCUP database and is weighted to generate nationally representative estimates.

We identified 149,220 discharges and 29,655 readmissions within 30 days involving the procedure of insertion, replacement, or removal of EVS by applying the International Classification of Disease, Ninth Edition, Clinical Modification (ICD-9-CM) and Clinical Classification Software (CCS) Procedure Code 2, which included ICD-9-CM Procedure Codes of 231-235, 239, 242 and 243. Data were extracted for years 2009 to 2013 [4]. Year-wise distributions of index stays, readmission, percent readmission, cost for index stays and cost for readmissions for patients requiring EVS procedures who possess Medicare insurance (ME-patients) and Medicaid insurance (MD-patients) were described.

Z-test statistic was used to compare the two groups. This study was conducted in compliance with the ethical standards of the responsible institution on human subjects as well as with the Helsinki Declaration. Our institution exempted this analysis from full review by the Institutional Review Board (HSC20150408N).

\section{Results}

During the 5 years of study, we recorded 149,220 index stays and 29,655 readmissions within 30 days involving the procedures of insertion, replacement, or removal of an EVS. The majority of patients $(27.92 \%)$ were between 18 and 44 years old, $51.34 \%$ of whom were males. Throughout the study period, hospital readmissions involving patients requiring procedures involving EVS consistently demonstrated both the highest annual mean cost for readmissions and the highest percentage of patient readmissions in regard to all neurosurgical procedures. The annual total index stays and the annual total readmissions for all patients requiring procedures involving EVS in the US did not vary significantly, with the number of index stays ranging from 31,820 to 28,546 , and the number of readmissions ranging from and 6,435 to 5,559 . There was an average of 29,844 index stays and 5,931 readmissions within 30 days (19.9\%) occurring annually during the study period (P $=0.1177, \mathrm{P}=0.0987)$. From 2009 to 2013 , the annual index stays and readmissions for ME-patients and MD-patient did not vary significantly, with the percentage of readmissions ranging from $19.2 \%$ to $17.5 \%$ and $21.2 \%$ to $21.8 \%$, respectively (Fig. $1, \mathrm{P}=0.197, \mathrm{P}=0.2594)$. However, as demonstrated by Figure 1 , the differences between the annual index stays and readmissions for ME-patients versus MD-patients requiring EVS were extremely statistically significant throughout the entire study period $(\mathrm{P}<0.0001, \mathrm{P}<0.0001)$. The mean cost for index stays for all patients requiring EVS procedures did not vary significantly from $\$ 36,811$ to $\$ 42,746$ with an average cost of $\$ 39,144$ for index stays occurring annually $(\mathrm{P}=0.1940)$; however, the mean cost of readmissions within 30 days for all patients varied significantly from $\$ 19,005$ to $\$ 23,499$, with an average cost of $\$ 21,279$ for readmissions occurring annually during the study period $(P=0.0161)$. From 2009 to 2013, the mean cost for index stays and readmissions for ME-patients varied significantly from $\$ 29,286$ to $\$ 34,150$ and $\$ 17,411$ to 
Table 1. Annual Mean Cost for Index Stays and Readmissions After EVS Procedures for Medicare and Medicaid Patients in US From 2009 to 2013

\begin{tabular}{|c|c|c|c|c|c|}
\hline & 2009 & 2010 & 2011 & 2012 & 2013 \\
\hline Annual mean cost of all index stays & $\$ 36,811$ & $\$ 37,864$ & $\$ 42,746$ & $\$ 39,472$ & $\$ 38,829$ \\
\hline Annual mean cost of all readmission & $\$ 19,005$ & $\$ 20,512$ & $\$ 21,076$ & $\$ 23,499$ & $\$ 22,303$ \\
\hline Annual mean cost of Medicare readmissions & $\$ 17,411$ & $\$ 19,856$ & $\$ 18,309$ & $\$ 19,522$ & $\$ 21,672$ \\
\hline Annual mean cost of Medicaid index stays & $\$ 39,924$ & $\$ 40,609$ & $\$ 47,726$ & $\$ 44,192$ & $\$ 43,003$ \\
\hline $\begin{array}{l}\text { P value for difference of index stays costs between } \\
\text { Medicare and Medicaid patients after EVS }\end{array}$ & $<0.0001$ & $<0.0001$ & $<0.0001$ & $<0.0001$ & $<0.0001$ \\
\hline $\begin{array}{l}\text { P value for difference of readmissions costs between } \\
\text { Medicare and Medicaid patients after EVS }\end{array}$ & $<0.0001$ & $<0.0001$ & $<0.0001$ & $<0.0001$ & $<0.0001$ \\
\hline
\end{tabular}

EVS: extracranial ventricular shunt.

$\$ 21,672$, with an average cost of $\$ 31,838$ and $\$ 19,354$ for index stays and readmissions, respectively (Table $1, \mathrm{P}=0.0278$, $\mathrm{P}=0.0032$ ). Additionally, throughout the study period, the mean cost for index stays for MD-patients did not vary significantly from $\$ 39,924$ to $\$ 47,726$, with an average cost of $\$ 41,090$ for index stays occurring annually $(\mathrm{P}=0.1571)$; however, the mean cost of readmissions within 30 days for MDpatients varied significantly from $\$ 19,856$ to $\$ 25,444$, with an average cost of $\$ 22,452$ for readmissions occurring annually, respectively (Table $1, \mathrm{P}=0.0244$ ). As demonstrated by Table 1 , the differences between the mean cost for index stays and readmissions for ME-patients versus MD-patients requiring EVS were extremely statistically significant throughout the entire study period $(\mathrm{P}<0.0001, \mathrm{P}<0.0001)$. As noted by Table 2 , the most frequent reason for readmission within 30 days after EVS was complication of a device, implant, or graft, which was the cause for readmission an average of $32.6 \%$ of the time throughout the study period.

\section{Discussion}

In this study, we found that 30-day readmission rates and mean cost of index stays and readmissions for patients re- quiring procedures involving EVS were consistently related to patients' payer insurance. Notably, there were significant differences between ME-patients and MD-patients in their respective annual index stays, readmission rates and mean costs for both indexes stays and readmissions, with healthcare costs involving MD-patients consistently being more expensive. These results prompt query into the discrepancies between patients' payer insurance and their outcomes after EVS procedures including rate of readmission, effects on neurosurgical economic trends, and possible implications of this information for patients and financial planners of healthcare institutions.

The etiology of the differences in 30-day readmissions and mean cost of index stays and readmissions between MEpatients and MD-patients may be multifactorial in nature. Previous studies have shown emergent index admission status and patient comorbidities were highly predictive of readmission $[5,6]$. Notably, Bina et al reported emergent admission more frequently for MD-patients compared with other primary payers, including ME-patients [5]. In addition, studies have shown that the severity of disease when admitted is highly predictive of readmission and that MD-patients were most frequently seen with extreme severity $[5,7]$. Similarly, Buchanan et al also analyzed readmissions within 30-days

Table 2. Most Frequent Reasons for Readmissions Within 30 Days After EVS Procedures for Patients in US From 2009 to $2013^{*}$

\begin{tabular}{|c|c|c|c|c|c|}
\hline & 2009 & 2010 & 2011 & 2012 & 2013 \\
\hline Complication of device, implant or graft & 34.3 & 32.8 & 30.7 & 33.2 & 32.2 \\
\hline Complications of surgical procedures or medical care & 5.5 & 6.2 & 6.0 & 4.0 & 6.5 \\
\hline Epilepsy, convulsions & - & - & 3.5 & 4.7 & - \\
\hline Other hereditary and degenerative nervous system conditions & 4.0 & - & 4.3 & 3.8 & \\
\hline Urinary tract infections & - & 3.8 & - & - & - \\
\hline Other nervous system disorders & 3.3 & - & - & - & - \\
\hline
\end{tabular}

${ }^{*}$ Percentage readmitted. EVS: extracranial ventricular shunt. 
of all their neurosurgical patients and identified a significant trend: the largest subgroup of their readmissions involved issues with CSF shunts [2]. Focusing on complications after CSF shunt procedures, Simon et al demonstrated many risk factors associated with shunt infection: young age, female sex, African American race, public insurance and subsequent revision procedures [8]. These results reflect similar findings from previous studies that have demonstrated that a large majority of readmissions after neurological surgery involve CSF shunts, with similar shunt failure rates throughout US, UK and Ireland [9-13]. These studies demonstrate that patient factors such as age and comorbidities, insurance status, and other factors contribute to the incidence of CSF shunt infections and may further influence the outcome discrepancies between ME-patients and MD-patients requiring EVS procedures. However, without further study of patient outcomes after EVS procedures, it is difficult to assess the true impact of the relationship between patients' payer insurance and their readmissions after EVS procedures.

Information involving patients' payer insurance and its effect on outcomes after EVS procedures may play an influential role in the financial aspects of neurosurgical treatment, which directly affects healthcare administrators of institutions. Previous investigation focusing on pediatric cases identified a statistically significant difference in the length of index visit stay between those with private insurance and those with public insurance, which may directly affect neurosurgical economic trends including mean cost of index stays [1]. Shannon et al reported a 1-day stay visit for those with public insurance; however, Walker et al reported that those with public insurance stayed 5 days longer than those with private insurance $[1,7]$. These findings suggest that a longer stay may be a contributing factor for the increased risk of readmission due to higher risk of infection, which is, overall, the most common reason for readmission after neurosurgical procedures $[12,13]$. Thus, a longer stay may also be a contributing factor to the higher cost of MD-patients' index stays. Additionally, Moghavem et al demonstrated that patients with Medicaid payer insurance are associated with increased risk of readmission, possibly suggesting low socioeconomic status correlates with higher health care spending [6]. Although there is a significant relationship between length of index stay and patient insurance status, the association between outcomes of patients undergoing EVS procedures and the effects of those outcomes on the financial aspects of neurosurgical procedures remains poorly understood and requires further investigation.

This study has several limitations that affect the interpretation of its results. The NIS does not provide clinical details such as vital statistics, laboratory data, or pharmacy data, and may have omitted potentially important confounding variables and details regarding medical treatments. The NIS utilizes large sample sizes, and we acknowledge the need for validation in additional datasets. We can only comment on the association of federal payer insurance and neurosurgical economic and readmission trends after EVS procedures since this is a retrospective analysis. Prospective studies would be needed to prove causation of payer insurance and neurosurgical trends after EVS procedures. Finally, generalizability of our study to countries other than the United States may not be possible as healthcare settings may differ.

\section{Conclusion}

Federal payer insurance has a significant association with neurosurgical economic and patient readmission trends after EVS procedures in hospitals in the US. Considering the significantly higher incidence of readmissions after EVS procedures compared to any other neurosurgical procedure, the outcomes of patients are significant for both patients and healthcare institutions. During the study period, MD-patients and ME-patients had consistently significantly different numbers of annual index stays, annual readmission rates, and mean costs for both indexes stays and readmissions. Specifically, for healthcare institutions, the financial burden and economics aspects of EVS procedures emphasize the importance of examining patient outcomes after these procedures, which have been demonstrated to be associated with patients' payer insurance. Further study is needed to investigate the etiology of these differences between patients' payer insurance and their impact on clinical outcomes after EVS procedures.

\section{Acknowledgments}

None to declare.

\section{Financial Disclosure}

None to declare.

\section{Conflict of Interest}

None to declare.

\section{Informed Consent}

Informed consent was not required by our institution for this study.

\section{Author Contributions}

RSE performed research including data collection, statistical analysis, created tables and figure and wrote the paper. MAE performed research including data collection, and wrote the paper. AS designed research and provided expert clinical knowledge to revise critically.

\section{Data Availability}

The data supporting the findings of this study have been deposited in HCUPnet database and can be accessed at https:// 
hcupnet.ahrq.gov/\#setup.

\section{References}

1. Shannon CN, Simon TD, Reed GT, Franklin FA, Kirby RS, Kilgore ML, Wellons JC, 3rd. The economic impact of ventriculoperitoneal shunt failure. J Neurosurg Pediatr. 2011;8(6):593-599.

2. Buchanan CC, Hernandez EA, Anderson JM, Dye JA, Leung M, Buxey F, Bergsneider M, et al. Analysis of 30day readmissions among neurosurgical patients: surgical complication avoidance as key to quality improvement. J Neurosurg. 2014;121(1):170-175.

3. Online HCUP overview course, in Health Cost and Utilization Project. http://www.hcup-us.ahrq.gov/overviewcourse.jsp. Accessed in January 2017.

4. Clinical Classifications Software (CCS) for ICD-9-CM Fact Sheet, in Health Cost and Utilization Project. https:// www.hcup-us.ahrq.gov/toolssoftware/ccs/ccsfactsheet. jsp. Accessed in January 2017.

5. Bina RW, Lemole GM, Dumont TM. Measuring quality of neurosurgical care: readmission is affected by patient factors. World Neurosurg. 2016;88:21-24.

6. Moghavem N, Morrison D, Ratliff JK, Hernandez-Boussard T. Cranial neurosurgical 30-day readmissions by clinical indication. J Neurosurg. 2015;123(1):189-197.
7. Walker CT, Stone JJ, Jain M, Jacobson M, Phillips V, Silberstein HJ. The effects of socioeconomic status and race on pediatric neurosurgical shunting. Childs Nerv Syst. 2014;30(1):117-122.

8. Simon TD, Hall M, Riva-Cambrin J, Albert JE, Jeffries HE, Lafleur B, Dean JM, et al. Infection rates following initial cerebrospinal fluid shunt placement across pediatric hospitals in the United States. Clinical article. J Neurosurg Pediatr. 2009;4(2):156-165.

9. Chern JJ, Bookland M, Tejedor-Sojo J, Riley J, Shoja MM, Tubbs RS, Reisner A. Return to system within 30 days of discharge following pediatric shunt surgery. J Neurosurg Pediatr. 2014;13(5):525-531.

10. Piatt JH, Jr. Pediatric spinal injury in the US: epidemiology and disparities. J Neurosurg Pediatr. 2015;16(4):463471.

11. Sarda S, Moore MK, Chern JJ. Letter to the editor: Readmission and reoperation after shunt surgery. J Neurosurg Pediatr. 2015;15(5):544.

12. Taylor BE, Youngerman BE, Goldstein H, Kabat DH, Appelboom G, Gold WE, Connolly ES, Jr. Causes and timing of unplanned early readmission after neurosurgery. Neurosurgery. 2016;79(3):356-369.

13. Kaur G, Stein LK, Boehme A, Liang JW, Tuhrim S, Mocco J, Dhamoon MS. Risk of readmission for infection after surgical intervention for intracerebral hemorrhage. J Neurol Sci. 2019;399:161-166. 